\title{
Expressão Protéica de p53 e c-myc como Marcadores no Prognóstico do Carcinoma de Colo Uterino
}

\author{
Autora: Sylvia Michelina Fernandes Brenna \\ Orientador: Prof. Dr. Luiz Carlos Zeferino \\ Co-Orientadora: Prof ${ }^{a}$. Dr ${ }^{\mathrm{a}}$. Glauce Aparecida Pinto
}

Tese de Doutorado apresentada ao Curso de Pós-Graduação da Faculdade de Ciências Médicas da Universidade Estadual de Campinas para obtenção do Título de Doutor em Tocoginecologia em 30 de junho de 2000.

Alguns estudos analisaram a expressão protéica de p53 e c-myc como marcadores no prognóstico do carcinoma de colo uterino, mas foram realizados em países desenvolvidos, onde há programa de rastreamento eficientes que podem selecionar estádios iniciais e formas agressivas da doença. O objetivo deste estudo foi analisar a associação destas proteínas com o prognóstico do carcinoma espino-celular (CEC) de colo uterino. Foi de coorte, incluiu 220 mulheres diagnosticadas entre 1992-1994, nos estádios Ib-III, seguidas por 5 anos. Os blocos de parafina foram processados para imuno-histoquímica: p53 (Dako DO7) e c-myc (Santa Cruz Biotechnology MX9E10). A análise baseou-se no teste de Cochran-Armitage, método de Kaplan-Meier, testes de Wilcoxon ou log-rank e modelo de risco proporcionais de Cox. A idade das mulheres variou de 2580 anos (média 53,4) e o CEC foi diagnosticado em
$22 \%, 28 \%$ e $50 \%$, nos estádios I, II e III respectivamente. A freqüência das proteínas foi $35 \%$ para p53 e $40 \%$ para c-myc. Houve tendência linear inversa entre a faixa etária e a proteína p53 $(\mathrm{p}=0,03)$ e entre o tempo de atividade sexual e a proteína $\mathrm{c}-\mathrm{myc}(\mathrm{p}=0,03)$. As mulheres no estádio II com proteína p53 positiva tiveram menor sobrevida livre de doença $(\mathrm{p}<0,01)$ e risco de recidiva 2,6 (IC 1,1-6,2) vezes maior. As mulheres com controle clínico da doença pós-tratamento com proteina $\mathrm{c}$-myc positiva tiveram menor sobrevida livre de doença $(\mathrm{p}<0,01)$ e risco de recidiva 2,1 (IC 1,1 $3,9)$ vezes maior. As proteinas p53 e c-myc associamse ao mau prognóstico do CEC nos primeiros 12 meses de seguimento.

Palavras-chave: Colo: câncer. Oncogenes.

\section{Associação entre História Clínica e Achados Urodinâmicos em Mulheres com Incontinência Urinária Transuretral}

\author{
Autor: Antônio Cardoso Pinto \\ Orientador: Prof. Dr. José Rafael Macéa
}

Dissertação de Mestrado apresentada ao Curso de pós-graduação em Cirurgia Geral da Faculdade de Ciências Médicas da Santa Casa de São Paulo em 20 de maio de 1999.

Com intuito de determinar a relação entre dados de história clínica e os diagnósticos obtidos em exame urodinâmico, estudamos 126 pacientes do sexo feminino, com história clínica de incontinência urinária trans-uretral, com cistoceles pequenas sem patologia infecciosa ou neurológica associada. Nas pacientes com história clínica de incontinência urinárias exclusiva por esforço obteve-se $87,5 \%$ de concordância com o diagnóstico urodinâmico, e nas pacientes em que a incontinência ao esforço era associada a urgência miccional encontramos apenas $55 \%$ de concordância no diagnóstico. Ao aplicarmos os testes estatísticos encontramos diferença significativa $(p<0,0001)$, o que justificaria a realização do estudo urodinâmico neste grupo de mulheres. Por outro lado não encontramos relação significativa entre os achados urodinâmicos e a faixa etária $(\mathrm{p}=0,331)$, cirurgias prévias para trata- mento de incontinência urinária genuína de esforço $(p=0,651)$ e menacme ou pós-menopausa $(p=0,627)$. Concluímos que: 1) a história clínica de perdas urinárias aos esforços exclusiva é de alta sensibilidade para o diagnóstico de incontinência urinária genuina de esforço. 2) A presença do sintoma urgência miccional associado a incontinência urinária por esforço é indicativo para realização do estudo urodinâmico, no intuito de prever doenças associadas, e propor o melhor tratamento. 3) A faixa etária, presença ou não de cirurgia prévia, bem como o estado fisiológico da paciente (menacme ou pós-menopausa) não influencia o diagnóstico urodinâmico.

Palavras-chave: Incontinência urinária. Estudo urodinâmico. 